\title{
Early and Long-term Effects of the Autologous Peripheral Stem Cell Implantation for Critical Limb Ischemia
}

\author{
Takahiro Nishida, MD, ${ }^{1}$ Yasutaka Ueno, MD, ${ }^{2}$ Takashi Kimura, MD,${ }^{2}$ Ryousuke Ogawa, MD, ${ }^{2}$ \\ Kunihiko Joo, MD, ${ }^{1}$ and Ryuji Tominaga, $\mathrm{MD}^{1}$
}

\begin{abstract}
Objective: Therapeutic angiogenesis by peripheral blood mononuclear cells (PB-MNCs) implantation has been shown to be a safe and effective treating for critical limb ischemia (CLI). We herein report our investigation of the long-term efficacy of implantation of granulocyte-colony stimulating factor (G-CSF)induced PB-MNCs to treat patients with CLI for which surgical bypass and/or percutaneous transluminal angioplasty are not possible.

Methods and Methods: Eleven cases were enrolled in this study. Following an injection of G-CSF (250 ug/day) for 3 days, PB-MNCs (1.1 $\pm 0.5 \times 10^{10}$ including $1.5 \pm 0.2 \times 10^{7}$ CD34-positive cells) were harvested by apheresis and then injected into 13 ischemic limbs.

Results: Resting pain either diminished or improved in 10 cases $(91 \%)$ at 4 weeks, and ulcer formation was cured in 6 out of $10 \mathrm{limbs}(60 \%)$ after treatment. The time required to enhance the arteries at the level of foot-joint by angiography which was performed in the abdominal aorta was shortened by 1 month $(10 \pm 4$ seconds) and 6 months $(12 \pm 1)$ compared with the pretreatment time $(15 \pm 5)$. Three patients died after treatment, and the actuarial survival rate at 3 years was $73 \%$. Freedom from major amputation at 3 years was $92 \%$.
\end{abstract}

Conclusion: The local injection of G-CSF-induced PB-MNCs showed striking early and long-term effects.

Keywords: peripheral blood mononuclear cells, arteriosclerosis obliterans, thromboangiitis obliterance, critical limb ischemia, angiogenesis

\section{INTRODUCTION}

Critical limb ischemia (CLI) due to arteriosclerosis obliterans (ASO) or thromboangiitis obliterance (TAO) is a disease that significantly decreases a patients' quality of life because of leg pain, ulceration, and gangrene.

\footnotetext{
${ }^{1}$ Department of Cardiovascular Surgery, Kyushu University, Fukuoka, Fukuoka, Japan

${ }^{2}$ Department of Cardiovascular Surgery, Shimonoseki City Hospital, Shimonoseki, Yamaguchi, Japan
}

Received: June 27, 2011; Accepted: September 23, 2011 Corresponding author: Takahiro Nishida, MD. Department of Cardiovascular Surgery, Kyushu University Graduate School of Medical Sciences, 3-1-1 Maidashi, Higashi-ku, Fukuoka, Fukuoka 812-8582, Japan

Tel: +81-92-642-5557, Fax: +81-92-642-5566

E-mail: tnishida@heart.med.kyushu-u.ac.jp
Although several therapies, including medical and surgical procedures, may reduce patients' symptoms and improve the condition of their legs, a lot of patients are not candidates for surgery or percutaneous transluminal angioplasty, and $25 \%$ of CLI patients requires a major amputation of a leg within 1 year after the first diagnosis of CLI. ${ }^{1)}$ Recently, a new therapy using bone marrow mononuclear cells (BM-MNCs) has been developed to treat CLI, and the implantation of granulocyte-stimulation factor (G-CSF)-derived peripheral blood mononuclear cells (PB-MNCs) into the ischemic limb have also been shown to be effective. ${ }^{2-4)}$ However, a few reports ${ }^{5)}$ of critical limb ischemia have, so far, examined the longterm ( $>3$ years) effect of PB-MNCs therapy for CLI. We herein report the acute and long-term effects of PB-MNCs therapy against CLI. 
Table 1 Profiles of the patients underwent PB-MNCs therapy

\begin{tabular}{lccccccc}
\hline Case & Gender & Age & Rest pain & Ulcer & DM & HD & Diagnosis \\
\hline $1(\mathrm{~L})$ & Male & 75 & + & - & + & + & ASO \\
$2(\mathrm{~L})$ & Female & 72 & + & + & + & - & ASO \\
$3(\mathrm{R})$ & Male & 69 & + & + & + & + & DM \\
$3(\mathrm{~L})$ & Male & 69 & + & + & + & + & DM \\
$4(\mathrm{~L})$ & Male & 70 & + & + & + & - & ASO \\
$5(\mathrm{~L})$ & Male & 79 & + & + & - & - & ASO \\
$6(\mathrm{R})$ & Female & 83 & + & + & + & + & ASO \\
$7(\mathrm{~L})$ & Male & 74 & + & - & - & - & ASO \\
$8(\mathrm{R})$ & Female & 82 & + & + & - & - & ASO \\
$9(\mathrm{R})$ & Female & 81 & + & + & + & - & TAO \\
$9(\mathrm{~L})$ & Female & 81 & + & + & + & - & TAO \\
$10(\mathrm{~L})$ & Male & 69 & + & - & - & - & ASO \\
$11(\mathrm{R})$ & Female & 83 & + & + & - & - & ASO \\
\hline
\end{tabular}

L: left lower limb; R: right lower limb; HD: hemodialysis; DM: diabetes mellitus; ASO: arteriosclerosis obliterans; TAO: thromboangiitis obliterans

\section{Materials ANd Methods}

Patients with CLI suffering from rest pain and/or ulcer formation on the limbs for at least three months, which did not improve despite possible bypass graft operation and/or medical treatment, were included in this study. All patients underwent angiography before this treatment, and 2 radiologists and 3 vascular surgeons evaluated whether PTA and/or bypass grafting was possible. When it was determined that it was impossible to perform PTA and/or bypass grafting, we then considered PB-MNCs therapy. Patients with severe ischemic heart disease, cerebrovascular disease, proliferative diabetic retinopathy, malignancy within one year, or who were unable to give fully informed consent, were excluded. After approval by the medical ethics committee of Shimonoseki City Hospital, 11 patients (13 legs) underwent the PBMNCs therapy during from December 2006 to May 2008. All patients underwent angiography before and after PB-MNCs therapy. Two radiologists carried out the angiographic examination of the lower body from the brachial artery and injected contrast medium into the abdominal aorta near the bifurcation. We measured the time from the injection of the contrast medium to the starting point of the enhancement of the artery at the level of the foot-joint. Characteristics of the patients are shown in Table 1. All patients were suffering from rest pain, and ulcer formation with and without gangrene were recognized in 10 legs of 8 patients. Six patients were suffering from diabetes mellitus (DM) and 3 patients underwent hemodialysis for chronic renal failure. Nine patients were suffering from arteriosclerosis obliterans (ASO), and the rest was suffering from DM angiopathy (case 3) or thromboangiitis obliterans (TAO; case 9). Previous treatments for patients are listed in the Table 2. All patients underwent medical treatment at least 3 months before PB-MNCs therapy. Two patients had already undergone major amputation in the contra-lateral limb. Patients with diabetic angiopathy and TAO and one patientwith ASO had only been treated medically before PB-MNCs therapy. The rest of the ASO patients had undergone PTA and/or bypass surgery before PB-MNCs therapy.

\section{Protocol for PB-MNCs Therapy}

Patients were injected with $250 \mu \mathrm{g}$ of G-CSF subcutaneously for 3 days, and the next day PB-MNCs were harvested by cell apheresis using a cell separator (COBE Spectra $^{\mathrm{TM}}$, GAMBRO BCT, Lakewood, USA), in a final suspension volume of approximately $50 \mathrm{ml}$. We harvested $1.1 \pm 0.5 \times 10^{10}$ total mononuclear cells, of which $1.4 \pm$ $0.5 \times 10^{7}$ were CD 34 positive cells. Within a few minutes after harvesting PB-MNCs, the collected stem cell suspension was divided into $0.5 \mathrm{ml}$ with a syringe and injected with a 26 gauge needle into $40-60$ points of the 
Table 2 Previous treatments of the patients underwent PB-MNCs therapy

\begin{tabular}{ll}
\hline Case & Previous treatment \\
\hline 1 & PTA to Lt. EIA. Graft replacement of Lt. EIA and Rt. FA. \\
2 & PTA and Bilateral FPAK bypass (twice). Amputation of the contra-lateral limb. \\
3 & Medication \\
4 & Lt. FPBK bypass. Amputation of the contra-lateral limb. \\
5 & PTA to Rt. CIA and EIA \\
6 & PTA to bilateral CIA and CFA \\
7 & FF bypass \\
8 & Resection of the gangrene of the Lt. 2nd finger. \\
9 & Medication \\
10 & Lt. FPBK bypass. PTA to Lt. ATA. \\
11 & Medication \\
\hline
\end{tabular}

PTA: percutaneous transluminal angioplasty; EIA: external iliac artery; FA: femoral artery; FPAK: femoro-popliteal above knee; FPBK: femoro-popliteal below knee; CIA: common iliac artery; CFA: common femoral artery; FF: femoro-femoral; ATA: anterior-tibial artery

Table 3 Results of ulcer after PB-MNCs therapy

\begin{tabular}{lcccccc}
\hline Case & Gender & Age & Ulcer & No. Size $(\mathrm{mm})$ & Improvement & Recurrence \\
\hline $2(\mathrm{~L})$ & Female & 72 & + & $2(22 \times 20,12 \times 8)$ & + & - \\
$3(\mathrm{R})$ & Male & 69 & + & $2(18 \times 10,10 \times 8)$ & + & - \\
$3(\mathrm{~L})$ & Male & 69 & + & $1(8 \times 8)$ & + & - \\
$4(\mathrm{~L})$ & Male & 70 & + & $1(5 \times 5)$ & + & - \\
$5(\mathrm{~L})$ & Male & 79 & + & $1(5 \times 3)$ & + & - \\
$6(\mathrm{R})$ & Female & 83 & + & $1(8 \times 5)$ & + & - \\
$8(\mathrm{R})$ & Female & 82 & + & $1(5 \times 5)$ & + & - \\
$9(\mathrm{R})$ & Female & 81 & + & $1(8 \times 5)$ & + & - \\
$9(\mathrm{~L})$ & Female & 81 & + & $1(10 \times 5)$ & + & + \\
$11(\mathrm{R})$ & Female & 83 & + & $1(5 \times 5)$ & + \\
\hline
\end{tabular}

L: left lower limb; R: right lower limb; No.: number

ischemic limb under venous anesthesia using midazolam $(0.5 \mathrm{mg} / \mathrm{kg})$. All patients were discharged from our hospital on the fifth day.

\section{RESUlts}

There were no significant side effects of this procedure during the five days of hospitalization. Ten (91\%) of 11 patients reported either diminished or deceased rest pain at 2 weeks after PB-MNCs therapy. Table 3 shows the number, size and fate of the ulcers after treatment. Ulcer formation was completely cured or improved in $8(80 \%)$ out of 10 legs at 4 weeks after PB-MNCs therapy, while no improvement of ulcer formation was observed in the other 2 cases during the follow-up period. Recurrences of ulcers were observed in 2 patients (Table 3). Thus, $60 \%$ of ulcers were cured without recurrence after PBMNCs therapy. Figure 1 shows an improvement of an ischemic ulcer in one patient (case 2 in Table 1). One patient died of pneumonia at 3 months after PB-MNCs therapy. This patient underwent a major amputation, 1 month after PB-MNCs therapy.

The ankle-brachial index (ABI) was improved from a baseline of $0.77 \pm 0.21$ to $0.91 \pm 0.27$ and $0.99 \pm 0.1$ at 1 


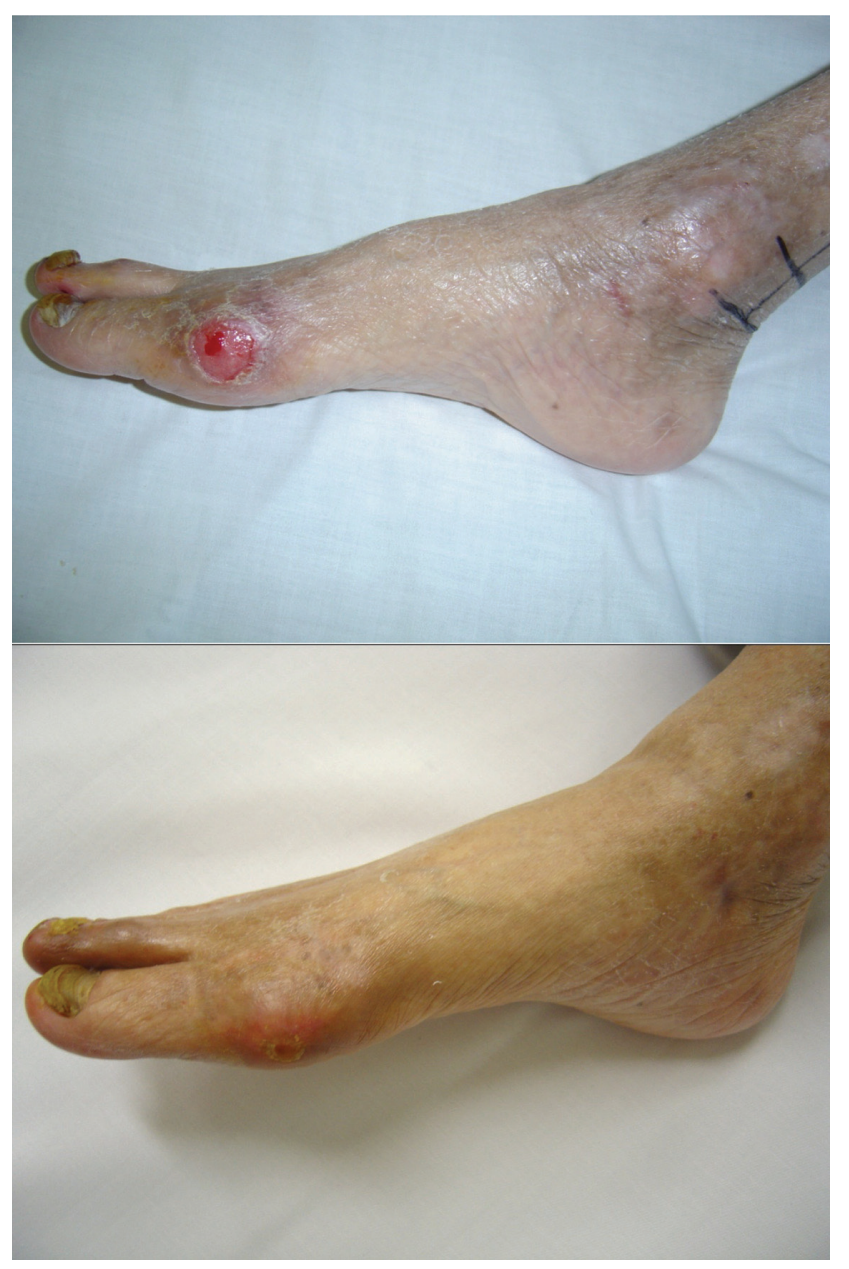

Fig. 1 Ulcer formation in case 2 (A) was completely healed after PB-MNCs therapy (B).

and 2 months after PB-MNCs therapy, respectively, and the ABI was still higher one $(0.84 \pm 0.28)$ and two years $(0.90 \pm 0.13)$ later. The time required to enhance the arteries at the level of the foot-joint by angiography was significantly shortened at 1 month $(10 \pm 4$ seconds, $\mathrm{p}<0.05)$ and 6 months $(12 \pm 1)$ after PB-MNCs therapy compared with the time of before treatment $(15 \pm 5$, Fig. 2).

The actuarial survival rate and freedom from major amputation of the lower limb at 36 months after PBMNCs therapy were $73 \%$ and $92 \%$, respectively (Fig. 3). The causes of death were a hepatic coma and sepsis in 2 patients (cases 1 and 6) and pneumonia (case 7) in 1 patient. Because no severe infection was observed in the lower limb of patients in a septic state, we did not carry out amputation of the lower limb. Both patients finally died of hepatic coma and septic shock due to necrosis of the small intestine.

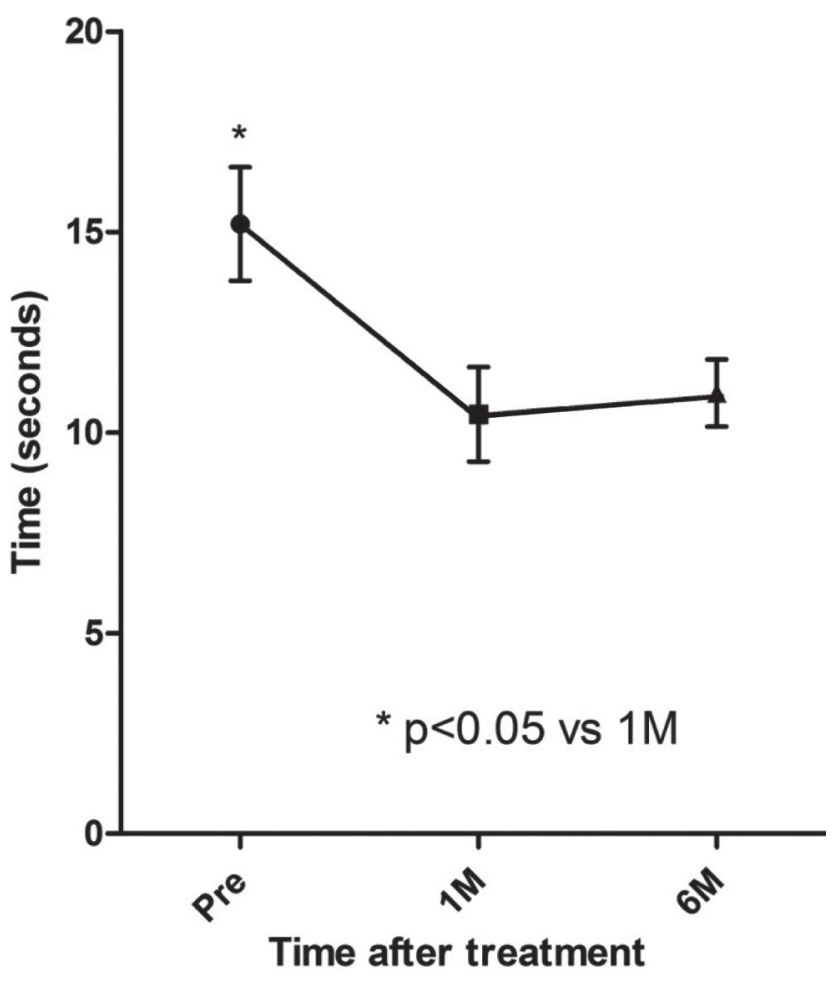

Fig. 2 The time required to enhance the arteries by angiography at the level of the foot-joint was significantly shortened at 1 month $(10 \pm 4$ seconds, $\mathrm{p}<0.05)$ after PB-MNCs therapy compared with the time of pretreatment $(15 \pm 5)$.

\section{Discussion}

In this report, we demonstrated the superior early and long-term results of PB-MNCs therapy for critical limb ischemia. Thanks to PB-MNCs therapy, rest pain was diminished or decreased in $91 \%$ of the patients and ischemic ulcer formation was cured without recurrence in $60 \%$ of the legs. The actuarial survival rate and freedom from major amputation of the lower limb at 36 months after PB-MNCs therapy were $73 \%$ and $92 \%$, respectively.

According to the ACC/AHA guidelines, ${ }^{1)}$ the prognosis of critical limb ischemia is relatively poor. Twenty-five percents of the patients require major amputation of the diseased limb, and only $50 \%$ of the patients will be alive with two limbs at one year after diagnosis of critical limb ischemia. It is, therefore, important to develop new treatment for CLI, especially those who are not indicated for surgery. Recently, angiogenetic therapeutic methods using bone marrow mononuclear cells (BM-MNCs) for CLI have been shown to have beneficial effects. ${ }^{6,7)}$ However, one-third of the treated patients did not respond 

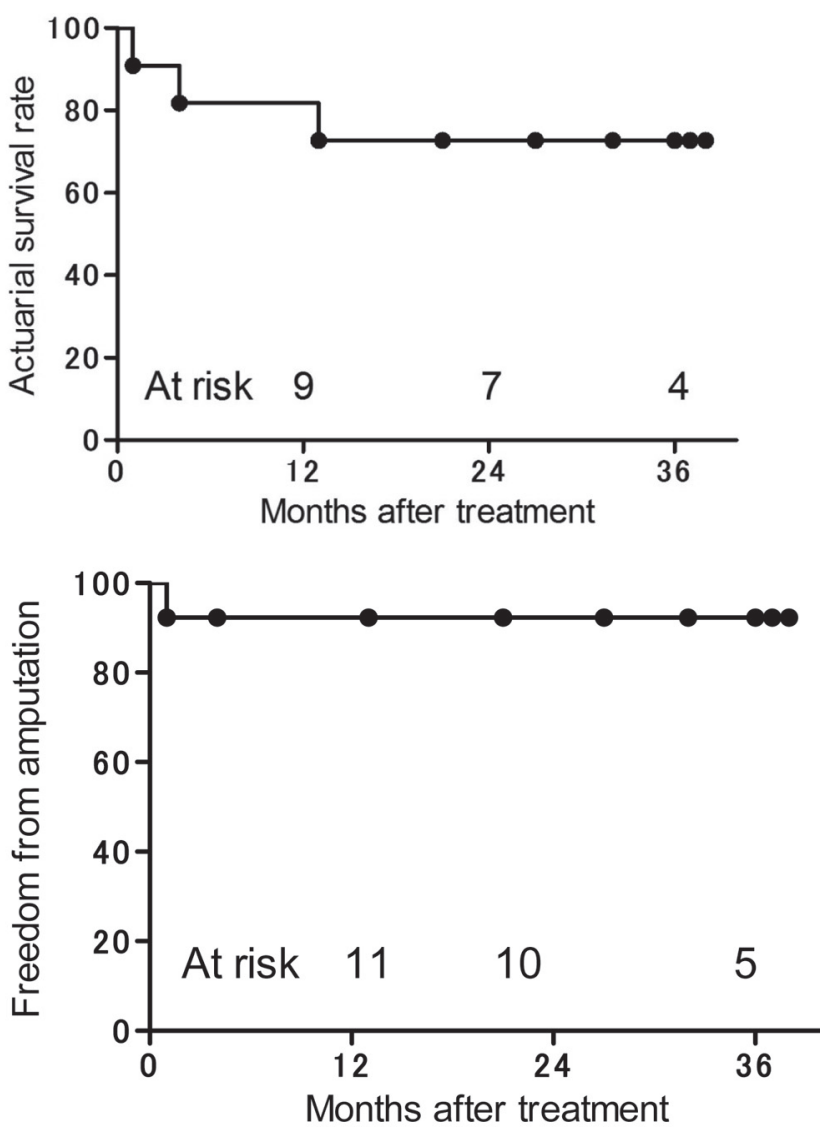

Fig. 3 Actuarial survival rate (A) and freedom from major amputation of the lower limb (B) at 36 months after PBMNCs therapy were $73 \%$ and $92 \%$.

well, and it is sometimes difficult to use BM-MNCs in the high-risk patients because general anesthesia is required to obtain a significant number of BM-MNCs. Therefore, less-invasive PB-MNCs therapy has been developed to treat CLI.

Hoshino et al. ${ }^{2)}$ described the efficacy of PB-MNCs therapy for CLI patients with diabetes mellitus on hemodialysis. Skin perfusion pressure, skin temperature, and ABI were maintained at a higher level than those at baseline during the 6 months after PB-MNCs therapy. Huang et al. ${ }^{3)}$ compared the efficacy between BM-MNCs therapy and PB-MNCs therapy to treat CLI. According to their study, comparative analysis revealed that at 12 weeks after cell implantation therapy, improvement of $\mathrm{ABI}$, skin temperature, and rest pain was significantly better in PB-MNCs patients than BM-MNCs patients. Although the exact explanation was not clear, the authors speculated that the role of G-CSF used in PB-MNCs therapy might have exerted some favorable effects to promote superior results, because G-CSF induced the production of several growth factors. Although other reports $^{8-10)}$ have also described the beneficial effects of PB-MNCs therapy, only a few reports have so far examined the long term effects of PB-MNCs therapy. Horie et al. ${ }^{5)}$ reported a 2 year survival rate of $65 \%$ and a 1 year amputation free rate of $70 \%$ in ASO patients after PBMNCs therapy.

We followed 11 patients for over 3 years and observed 3 deaths and one major amputation after PB-MNCs therapy. Two patients among the 3 dead patients underwent hemodialysis for end-stage renal failure. Although one patient died of sepsis at 4 months after treatment, and the other died of a hepatic coma at 12 months after the treatment. One patient underwent major amputation, 2 months after the PB-MNCs therapy, and later died of severe pneumonia. This was the only patient who required major amputation after PB-MNCs therapy, and the remaining 10 patients have not undergone any major amputation, indicating a superior amputation free ratio of $92 \%$ at three years after PB-MNCs therapy. We also showed that the time required to enhance the arteries by angiography at the level of the foot-joint was significantly shortened at 1 month after PB-MNCs therapy. Although it is difficult to clarify the exact mechanism responsible for these superior long term effects, once improved microcirculation induced by PB-MNCs therapy might continue to enhance the favorable effects for local circulation of the ischemic limbs.

Our study has, therefore, demonstrated that G-CSFinduced PB-MNCs therapy showed striking early and long term results, however, because of the relatively small number of patients enrolled this study, more patients and a longer follow-up duration will be required to confirm our data.

\section{REFERENCES}

1) Hirsch AT, Haskal ZJ, Hertzer NR, et al. ACC/AHA 2005 Practice Guidelines for the management of patients with peripheral arterial disease (lower extremity, renal, mesenteric, and abdominal aortic): a collaborative report from the American Association for Vascular Surgery/Society for Vascular Surgery, Society for Cardiovascular Angiography and Interventions, Society for Vascular Medicine and Biology, Society of Interventional Radiology, and the ACC/AHA Task Force on Practice Guidelines (Writing Committee to Develop Guidelines for the Management of Patients With Peripheral Arterial Disease): endorsed by the American Association of Cardiovascular and Pulmonary 
Rehabilitation; National Heart, Lung, and Blood Institute; Society for Vascular Nursing; TransAtlantic Inter-Society Consensus; and Vascular Disease Foundation. Circulation 2006; 113: e463-654 Review. [Medline] [CrossRef]

2) Hoshino J, Ubara Y, Hara S, et al. Quality of life improvement and long-term effects of peripheral blood mononuclear cell transplantation for severe arteriosclerosis obliterans in diabetic patients on dialysis. Circ J 2007; 71: 1193-8. [Medline] [CrossRef]

3) Huang PP, Yang XF, Li SZ, et al. Randomised comparison of G-CSF-mobilized peripheral blood mononuclear cells versus bone marrow-mononuclear cells for the treatment of patients with lower limb arteriosclerosis obliterans. Thromb Haemost 2007; 98: 133542. [Medline]

4) Kajiguchi M, Kondo T, Izawa H, et al. Safety and efficacy of autologous progenitor cell transplantation for therapeutic angiogenesis in patients with critical limb ischemia. Circ J 2007; 71: 196-201. [Medline] [CrossRef]

5) Horie T, Onodera R, Akamastu M, et al. Long-term clinical outcomes for patients with lower limb ischemia implanted with G-CSF-mobilized autologous peripheral blood mononuclear cells. Atherosclerosis 2010;
208: 461-6 Epub 2009 Aug 3. [Medline] [CrossRef]

6) Tateishi-Yuyama E, Matsubara H, Murohara T, et al. Therapeutic angiogenesis for patients with limb ischaemia by autologous transplantation of bone-marrow cells: a pilot study and a randomised controlled trial. Lancet 2002; 360: 427-35. [Medline] [CrossRef]

7) Yamamoto K, Kondo T, Suzuki S, et al. Molecular evaluation of endothelial progenitor cells in patients with ischemic limbs: therapeutic effect by stem cell transplantation. Arterioscler Thromb Vasc Biol 2004; 24: e192-6 Epub 2004 Oct 14. [Medline] [CrossRef]

8) Huang P, Li S, Han M, et al. Autologous transplantation of granulocyte colony-stimulating factor-mobilized peripheral blood mononuclear cells improves critical limb ischemia in diabetes. Diabetes Care 2005; 28: 2155-60. [Medline] [CrossRef]

9) Ishida A, Ohya Y, Sakuda H, et al. Autologous peripheral blood mononuclear cell implantation for patients with peripheral arterial disease improves limb ischemia. Circ J 2005; 69: 1260-5. [Medline] [CrossRef]

10) Zhou B, Bi YY, Han ZB, et al. G-CSF-mobilized peripheral blood mononuclear cells from diabetic patients augment neovascularization in ischemic limbs but with impaired capability. J Thromb Haemost 2006; 4: 993 1002. [Medline] [CrossRef] 